TRANSACTIONS OF THE

AMERICAN MATHEMATICAL SOCIETY

Volume 348, Number 8, August 1996

\title{
THE SCHWARZIAN DERIVATIVE FOR MAPS BETWEEN MANIFOLDS WITH COMPLEX PROJECTIVE CONNECTIONS
}

\author{
ROBERT MOLZON AND KAREN PINNEY MORTENSEN
}

\begin{abstract}
In this paper we define, in two equivalent ways, the Schwarzian derivative of a map between complex manifolds equipped with complex projective connections. Also, a new, coordinate-free definition of complex projective connections is given. We show how the Schwarzian derivative is related to the projective structure of the manifolds, to projective linear transformations, and to complex geodesics.
\end{abstract}

\section{INTRODUCTION}

In our paper [13], we defined and studied two operators, $\Sigma$ and $L$, associated to a locally biholomorphic mapping from a domain in $\mathbf{C}^{n}$ to $\mathbf{C} P^{n}$. These two operators were shown to play a role analogous to the Schwarzian derivative $\left(f^{\prime \prime} / f^{\prime}\right)^{\prime}-\frac{1}{2}\left(f^{\prime \prime} / f^{\prime}\right)^{2}$ for functions of one complex variable; in particular, a geometric interpretation of $\Sigma$ allowed us to give a simple proof that a mapping is complex projective linear if and only if $\Sigma$ vanishes identically.

In this paper we generalize these ideas by defining a Schwarzian derivative $\Sigma_{\phi}$ for any locally biholomorphic map $\phi: M \rightarrow N$ where $M$ and $N$ are complex manifolds of dimension $n$, each with a fixed complex projective connection. $\Sigma_{\phi}$ is a symmetric tensor on $M$ which measures the extent to which $\phi$ does not preserve the complex projective connection; it is essentially the difference between the projective connections. It is of interest to compare $\Sigma_{\phi}$ with the Schwarzian derivative which Osgood and Stowe defined for a conformal map between Riemannian manifolds [15]. They showed that their Schwarzian derivative can be defined as a difference of two "Möbius connections" [14]. Also, Deligne showed that for holomorphic functions of one variable, the classical Schwarzian derivative can be given as a difference of projective connections [2].

We include a fairly complete discussion of complex projective connections. The projective connection is an old and well-developed notion in the setting of real manifolds. It has been carried over to complex manifolds in [10], [8], [6], [4], and others. In Section 4 we give a new definition of a complex projective connection. The connection need not be holomorphic, but if it is, then this definition is equivalent to that of [10]. Our definition has the advantage of being coordinate-free. Although not every complex manifold supports a holomorphic projective connection [6], [7], a study of the relationship between affine connections and projective

Received by the editors August 15, 1994.

1991 Mathematics Subject Classification. Primary 53B10, 32H02.

The second author was partially supported by NSF grant DMS-9102976.

(C)1996 American Mathematical Society 
connections shows that every complex manifold supports a complex projective connection (Corollary 4.1).

In Section 5 we show how principal bundles and Cartan connections may be used to define holomorphic projective connections. This approach was used to study projective connections on differentiable manifolds in [1] and [9]. Ganesh [4] extended the principal bundle approach to study holomorphic projective connections on complex manifolds, but did not use the idea of a Cartan connection. To complete this circle of ideas, we define a "normal complex projective Cartan connection" and show that it coincides with the complex projective connection defined in Section 4 . The relationship is given explicitly in Proposition 5.3.

We give two equivalent definitions of the Schwarzian derivative $\Sigma_{\phi}$. From the first definition, given in Section 6 , it is easy to see that when the projective connections on $M$ and $N$ are flat, then $\Sigma_{\phi}$ generalizes the operator $\Sigma$ described in [13]. The second definition is considerably more complicated, but it gives a valuable geometric interpretation of $\Sigma_{\phi}$. This method is inspired by the work of Thomas (for real manifolds) [18] and of Flanders (for functions of one complex variable) [3]. In order to give this second definition, we construct in Section 7 a line bundle $\hat{M}$ over $M$. If $M$ has a complex projective atlas, then $\hat{M}$ has a complex affine atlas (Proposition 7.1). Furthermore, $\hat{M}$ is endowed with a canonical volume form and to each complex projective connection on $M$ is associated an affine connection on $\hat{M}$ (Theorem 7.1). In Section 8 we define the Schwarzian derivative of $\phi: M \rightarrow N$ by using the affine connections on $\hat{M}$ and $\hat{N}$ and a canonical lift $\hat{\phi}: \hat{M} \rightarrow \hat{N}$ of the map $\phi$. This generalizes a construction given in [13] for maps from a domain in $\mathbf{C}^{n}$ to $\mathbf{C} P^{n}$. If the projective connections on $M$ and $N$ are flat, then this definition of $\Sigma_{\phi}$ leads to a geometric proof that $\Sigma_{\phi} \equiv 0$ if and only if $\phi$ is complex projective linear when written in projective coordinates (Theorem 8.1).

Projective geometry was conceived by Weyl [20] and by Thomas [17] as the geometry of "paths", that is, of unparametrized geodesics. Thomas obtained a projective connection from any class of affine connections which have the same (unparametrized) geodesics, and defined projective maps as those which preserve the geodesics. In Section 9, we show that these ideas may be applied to the holomorphic setting as well. In particular, we obtain Theorem 9.1, which says that a locally biholomorphic map $\phi$ between complex manifolds with reduced holomorphic projective connections preserves complex geodesics if and only if $\Sigma_{\phi} \equiv 0$.

We are grateful to Robin Graham and to Brad Osgood for several useful conversations.

\section{Preliminaries}

Let $M$ be a complex manifold of dimension $n$. The complex structure is given by an operator $J: T_{\mathbf{C}} M \rightarrow T_{\mathbf{C}} M ; T^{h} M$ denotes the $i$-eigenspace of $J$. Vector fields which are differentiable sections of $T^{h} M$ are called $(1,0)$-vector fields; the space of all $(1,0)$-vector fields is denoted by $\mathfrak{X}^{h} M$. The dual bundle to $T^{h} M$ is denoted $T^{h *} M$.

Throughout the paper, all affine connections are assumed to be torsion-free and compatible with the complex structure on $M$. All manifolds are assumed to be connected.

For every open covering of $M$, there is a refinement consisting of simply connected, Stein domains, all of whose intersections are also simply connected. We 
will assume, when necessary, that a covering of $M$ has these properties, without explicit mention.

We use the Einstein summation convention when working in local coordinates: one sums over all repeated indices. Unless otherwise specified, small Roman indices run from 1 to $n$ and capital indices run from 0 to $n$. The Kronecker delta $\delta_{I}^{J}$ equals 1 if $I=J, 0$ otherwise.

In this paper, "differentiable" will always mean $C^{\infty}$.

\section{Complex Projective Structures}

Let $M$ be a complex manifold of dimension $n$.

Definition 3.1. A complex projective atlas for $M$ is an atlas of coordinate charts covering $M$ such that all the coordinate changes are complex projective linear.

That is, if $\left(x_{\alpha}^{1}, \ldots, x_{\alpha}^{n}\right)$ are holomorphic coordinates on $U_{\alpha}$ and $\left(x_{\beta}^{1}, \ldots, x_{\beta}^{n}\right)$ are holomorphic coordinates on $U_{\beta}$, then on $U_{\alpha} \cap U_{\beta}$,

$$
x_{\beta}^{i}=f_{\alpha \beta}^{i}\left(x_{\alpha}\right)=\frac{a_{j}^{i} x_{\alpha}^{j}+b^{i}}{c_{j} x_{\alpha}^{j}+d},
$$

where $a_{j}^{i}, b^{i}, c_{j}$, and $d$ are complex constants and the determinant of the $(n+1) \times$ $(n+1)$ matrix $\left(\begin{array}{ll}a_{j}^{i} & b^{i} \\ c_{j} & d\end{array}\right)$ is nonzero. The coordinate charts in the atlas are called projective coordinates.

A complex projective atlas determines a 1-cocycle with values in the projective linear group $P L(n, \mathbf{C})$, and thus an element of $H^{1}(M, P L(n, \mathbf{C}))$.

Definition 3.2. Two projective atlases are said to be equivalent if they determine the same element of $H^{1}(M, P L(n, \mathbf{C}))$; the equivalence class is called a complex projective structure on $M$.

Some simple examples of complex manifolds which admit a complex projective structure are complex projective space and any manifold which can be expressed as a quotient of $\mathbf{C}^{n}$ or of complex hyperbolic space by a group of complex projective linear transformations. In [5], Gunning showed that every Riemann surface admits a complex projective structure. For dimensions greater than 1, there are obstructions to the existence of a complex projective structure. In [10], Kobayashi and Ochiai completely classified compact complex surfaces which admit a complex projective structure. For higher dimensions, examples of manifolds admitting and not admitting complex projective structures can be found in [10] and [8].

We recall the definition of the Schwarzian derivative in several complex variables. Let $U$ be an open set in $\mathbf{C}^{n}(n>1)$ and $f: U \rightarrow \mathbf{C}^{n}$ locally biholomorphic. Let $z=f(x)$ and let $J_{f}$ denote the Jacobian determinant $\operatorname{det}\left(\frac{\partial f^{i}}{\partial x^{j}}\right)$.

Definition 3.3. The Schwarzian derivative of $f$ is the tensor

$$
\begin{aligned}
S(f) & =S(f)_{i j}^{k} d x^{i} \otimes d x^{j} \otimes \frac{\partial}{\partial x^{k}} \\
& =\left(\frac{\partial^{2} z^{l}}{\partial x^{i} \partial x^{j}} \frac{\partial x^{k}}{\partial z^{l}}-\frac{\delta_{j}^{k}}{n+1} \frac{\partial \log J_{f}}{\partial x^{i}}-\frac{\delta_{i}^{k}}{n+1} \frac{\partial \log J_{f}}{\partial x^{j}}\right) d x^{i} \otimes d x^{j} \otimes \frac{\partial}{\partial x^{k}} .
\end{aligned}
$$


The Schwarzian derivative has appeared frequently in the literature, although not always by that name. See, for example, [4, p.71], [22, p.474], [23, p.128], [8, p.38], $[10$, p.76], and [6]. It is well known that $S(f)$ vanishes identically if and only if $f$ is complex projective linear; see pages 48-50 of Gunning's book [6]. A geometric proof

can be found in [13]. For $n=1$, the classical Schwarzian derivative $\left(\frac{f^{\prime \prime}}{f^{\prime}}\right)^{\prime}-\frac{1}{2}\left(\frac{f^{\prime \prime}}{f^{\prime}}\right)^{2}$ characterizes projective linear functions. Notice that it is a differential operator of order 3 , whereas $S(f)$ is of order 2 . In this paper we deal primarily with the case $n>1$, although some of the ideas apply to $n=1$ also.

\section{Complex Projective Connections}

Closely related to the idea of a complex projective structure is the notion of a complex projective connection. Differentiable projective connections on real manifolds were defined and studied in the 1920's by E. Cartan, and in a somewhat different guise, by T.Y. Thomas [1], [17]. The 1931 paper of J.H.C. Whitehead [21] recounts the early history of the subject.

In the complex setting, and in more modern language, holomorphic projective connections are described by Kobayashi and Ochiai in [10] and by Kobayashi and Horst in [8]. The definition for dimension $n=1$ can be found in [5] or in [19].

The definitions mentioned above are all in terms of local coordinates. In this section we give an equivalent coordinate-free definition and also define the more general notion of a complex projective connection. We also show how projective connections are related to complex projective structures and to classes of projectively equivalent affine connections.

Throughout this section, $M$ will denote a complex manifold of dimension $n>1$.

Definition 4.1. A complex projective connection on $M$ is a differentiable map

$$
\Pi: \mathfrak{X}^{h} M \times \mathfrak{X}^{h} M \rightarrow \mathfrak{X}^{h} M
$$

which satisfies

1.

$$
\Pi_{\left(X_{1}+X_{2}\right)} Y=\Pi_{X_{1}} Y+\Pi_{X_{2}} Y
$$

2.

$$
\Pi_{X}\left(Y_{1}+Y_{2}\right)=\Pi_{X} Y_{1}+\Pi_{X} Y_{2}
$$

3.

$$
\Pi_{(f X)} Y=f \Pi_{X} Y-\frac{1}{n+1} X(f) Y \quad \text { for } f \in C^{\infty}(M),
$$

4.

$$
\Pi_{X}(f Y)=f \Pi_{X} Y+X(f) Y-\frac{1}{n+1} Y(f) X \quad \text { for } f \in C^{\infty}(M),
$$

5.

$$
\Pi_{X} Y-\Pi_{Y} X=[X, Y] .
$$

The terminology regarding projective connections is not consistent in the literature. For example, Kato [7] omits property 5 from the definition and calls a projective connection normal if it satisfies property 5 . 
Definition 4.2. A reduced projective connection is a projective connection with the property that for any local holomorphic coordinates,

$$
d x^{i}\left(\Pi_{\frac{\partial}{\partial x^{i}}} \frac{\partial}{\partial x^{j}}\right)=0 \text { for all } j .
$$

A simple calculation shows that if for some local holomorphic coordinates

$$
d x^{i}\left(\Pi_{\frac{\partial}{\partial x^{i}}} \frac{\partial}{\partial x^{j}}\right)=0,
$$

then the same holds for all local holomorphic coordinates. Given a complex projective connection $\Pi$, one may construct a reduced complex projective connection $\tilde{\Pi}$ by setting

$$
\tilde{\Pi}_{\frac{\partial}{\partial x^{i}}} \frac{\partial}{\partial x^{j}}=\Pi_{\frac{\partial}{\partial x^{i}}} \frac{\partial}{\partial x^{j}}-\frac{1}{n} d x^{l}\left(\Pi_{\frac{\partial}{\partial x^{l}}} \frac{\partial}{\partial x^{j}}\right) \frac{\partial}{\partial x^{i}} .
$$

Definition 4.3. A holomorphic projective connection on $M$ is a complex projective connection $\Pi$ with the property that if $X$ and $Y$ are holomorphic vector fields (i.e. holomorphic sections of $\left.T^{h} M\right)$ near $p \in M$ and if $\theta$ is a holomorphic (1,0)-form near $p$, then the function $\theta\left(\Pi_{X} Y\right)$ is holomorphic near $p$.

We now recall the definition of holomorphic projective connection given by Kobayashi and Ochiai in [10] and show that it coincides with our definition.

Given any coordinate covering of a complex manifold $M$, let $x_{\beta}=f_{\alpha \beta}\left(x_{\alpha}\right)$ denote the coordinate transformations. Then $S=\left\{S\left(f_{\alpha \beta}\right)\right\}$ is a holomorphic 1cocycle with values in $T^{h *} M \otimes T^{h *} M \otimes T^{h} M$ and thus defines a cohomology class $[S]$ in $H^{1}\left(M, T^{h *} M \otimes T^{h *} M \otimes T^{h} M\right)$. This cohomology class is independent of the choice of coordinates. It is called by Kato ([7]) "the obstruction to the holomorphic projective connection."

Definition 4.4 ([10]). A holomorphic projective connection on $M$ is a holomorphic 0 -cochain $\Psi=\left\{\Psi_{\alpha}\right\}$ with values in $T^{h *} M \otimes T^{h *} M \otimes T^{h} M$ such that

(1) the coboundary of $\Psi$ is $S$,

(2) $\Psi$ is symmetric: for all $X, Y \in T^{h} M, \Psi_{\alpha}(X, Y)=\Psi_{\alpha}(Y, X)$.

In local coordinates, we write

$$
\Psi_{\alpha}=\Psi_{\alpha i j}^{k} d x_{\alpha}^{i} \otimes d x_{\alpha}^{j} \otimes \frac{\partial}{\partial x_{\alpha}^{k}} .
$$

Then condition (1) is equivalent to

$$
\Psi_{\alpha i j}^{k}=\frac{\partial x_{\beta}^{l}}{\partial x_{\alpha}^{i}} \frac{\partial x_{\beta}^{m}}{\partial x_{\alpha}^{j}} \Psi_{\beta l m}^{p} \frac{\partial x_{\alpha}^{k}}{\partial x_{\beta}^{p}}+S\left(f_{\alpha \beta}\right)_{i j}^{k}
$$

and condition (2) is equivalent to $\Psi_{\alpha i j}^{k}=\Psi_{\alpha j i}^{k}$.

Given a holomorphic projective connection $\Psi$ in the sense of Definition 4.3, we let

$$
\Pi_{\frac{\partial}{\partial x_{\alpha}^{i}}} \frac{\partial}{\partial x_{\alpha}^{j}}=\Psi_{\alpha}\left(\frac{\partial}{\partial x_{\alpha}^{i}}, \frac{\partial}{\partial x_{\alpha}^{j}}\right)
$$

and define $\Pi_{f^{i} \partial / \partial x_{\alpha}^{i}} g^{j} \frac{\partial}{\partial x_{\alpha}^{j}}$ using properties 1 through 4 of Definition 4.1. A direct computation, using the symmetry of $\Psi$, shows that property 5 also holds. Another 
direct computation, using properties 1 through 4 and the fact that

shows that

$$
\frac{\partial^{2} x_{\beta}^{l}}{\partial x_{\alpha}^{i} \partial x_{\alpha}^{p}} \frac{\partial x_{\alpha}^{p}}{\partial x_{\beta}^{l}}=\frac{\partial \log J_{f_{\alpha \beta}}}{\partial x_{\alpha}^{i}}
$$

$$
\Pi_{\frac{\partial}{\partial x_{\alpha}^{i}}} \frac{\partial}{\partial x_{\alpha}^{j}}=\frac{\partial x_{\beta}^{l}}{\partial x_{\alpha}^{i}} \frac{\partial x_{\beta}^{m}}{\partial x_{\alpha}^{j}} \Pi_{\frac{\partial}{\partial x_{\beta}^{l}}} \frac{\partial}{\partial x_{\beta}^{m}}+S\left(f_{\alpha \beta}\right)_{i j}^{k} \frac{\partial}{\partial x_{\alpha}^{k}} .
$$

Together with the transformation law (1), this shows that $\Pi$ is independent of the choice of $\alpha$.

Conversely, given $\Pi$, we may obtain $\Psi$ by setting

$$
\Psi_{\alpha i j}^{k}=d x_{\alpha}^{k}\left(\Pi_{\frac{\partial}{\partial x_{\alpha}^{i}}} \frac{\partial}{\partial x_{\alpha}^{j}}\right) .
$$

Henceforth we will write $\Pi_{\alpha i j}^{k}$ in place of $\Psi_{\alpha i j}^{k}$ and use the two definitions of holomorphic projective connection interchangeably.

Definition 4.5 ([10]). A holomorphic projective connection is called flat (or integrable) if there exists an atlas of holomorphic coordinate charts such that $\Pi_{\alpha i j}^{k} \equiv 0$ for all $\alpha$, for all $i, j, k$.

Theorem 4.1 ([10], [4]). A complex manifold $M$ has a flat holomorphic projective connection if and only if it has a complex projective structure.

Proof. If $M$ has a flat holomorphic connection, one may choose an atlas of holomorphic coordinate charts for which $\Pi_{\alpha i j}^{k}=0$ for all $\alpha$. The transformation law (1) then implies that the Schwarzian derivative of each coordinate transformation vanishes, so the coordinate transformations are complex projective linear.

Conversely, if $M$ has a complex projective structure, then with respect to a complex projective atlas, $S\left(f_{\alpha \beta}\right)=0$ for all $\alpha, \beta$, so $\Pi_{\alpha i j}^{k} \equiv 0$ defines a flat holomorphic projective connection.

Flat holomorphic projective connections are also characterized by the vanishing of the "Weyl projective curvature tensor"; see [6, pp.79-83].

For a differentiable (real) manifold, Weyl [20] defined a projective structure to be an equivalence class of differentiable affine connections. Two connections are equivalent if they define, up to reparametrization, the same geodesics. Thomas [17], [18] showed how such an equivalence class determines a (real) projective connection. We conclude this section by showing how these ideas carry over to the holomorphic setting. Recall that all connections are assumed to be compatible with the fixed complex structure.

Definition 4.6. Two affine connections $\nabla$ and $\nabla^{\prime}$ on a complex manifold $M$ are projectively equivalent if there exists a $(1,0)$-form $\omega$ such that for all $X \in T^{h} M$, $Y \in \mathfrak{X}^{h} M$,

$$
\nabla_{X}^{\prime} Y-\nabla_{X} Y=\omega(X) Y+\omega(Y) X
$$

By a "complex geodesic" we will mean a totally geodesic 1-dimensional complex submanifold $G$ of $M$. Let $B$ be a domain in the complex plane, $g: B \rightarrow G$ a local coordinate chart for $G, V=g_{*} \frac{\partial}{\partial z}$.

Lemma 4.1. The condition that $G$ be totally geodesic is equivalent to the condition that $\nabla_{V} V$ be a multiple of $V$ at every point of $g(B)$. 
Proof. Let $\zeta$ and $\tau$ be real vector fields tangent to $G$. This means $\zeta=a V+\bar{a} \bar{V}$, $\tau=b V+\bar{b} \bar{V} . G$ is totally geodesic if and only if $\nabla_{\zeta} \tau$ is also tangent to $G$. Since $V$ is holomorphic (i.e. has holomorphic coefficients when written in local coordinates), $\nabla_{V} \bar{V}=\nabla_{\bar{V}} V=0$. Therefore

$$
\nabla_{\zeta} \tau=[a V(b)+\bar{a} \bar{V}(b)] V+[\bar{a} \bar{V}(\bar{b})+a V(\bar{b})] \bar{V}+a b \nabla_{V} V+\bar{a} \bar{b} \nabla_{\bar{V}} \bar{V},
$$

which is tangent to $G$ if and only if $\nabla_{V} V$ is a multiple of $V$.

Theorem 4.2. Two affine connections $\nabla$ and $\nabla^{\prime}$ on a complex manifold $M$ are projectively equivalent if and only if they determine the same complex geodesics.

This is a corollary of the more general Theorem 9.1 proved in Section 9; we defer the proof until then.

The proof of the next theorem is taken from ideas of Thomas [17] and Kobayashi and Ochiai [10].

Theorem 4.3. On a complex manifold, there is a one-to-one correspondence between reduced complex projective connections and projective equivalence classes of affine connections.

Proof. Let $\nabla$ be an affine connection on $M$. For $X \in \mathfrak{X}^{h} M$, let

$$
\eta(X)=\operatorname{tr}\left(Y \mapsto \nabla_{Y} X\right) .
$$

Define

$$
\Pi_{X} Y=\nabla_{X} Y-\frac{1}{n+1} \eta(X) Y-\frac{1}{n+1} \eta(Y) X .
$$

It is a straightforward computation to verify that $\Pi$ is a reduced complex projective connection. If $\nabla^{\prime}$ is projectively equivalent to $\nabla$, so that

$$
\nabla_{X}^{\prime} Y=\nabla_{X} Y+\omega(X) Y+\omega(Y) X,
$$

then

$$
\eta^{\prime}(X)=\operatorname{tr}\left(Y \mapsto \nabla_{Y}^{\prime} X\right)=\eta(X)+(n+1) \omega(X),
$$

so

$$
\nabla_{X}^{\prime} Y-\frac{1}{n+1} \eta^{\prime}(X) Y-\frac{1}{n+1} \eta^{\prime}(Y) X=\nabla_{X} Y-\frac{1}{n+1} \eta(X) Y-\frac{1}{n+1} \eta(Y) X .
$$

Therefore every affine connection which is projectively equivalent to $\nabla$ determines the same complex projective connection $\Pi$.

If, on the other hand, we begin with a reduced complex projective connection $\Pi$, we can construct from it an equivalence class of affine connections. To do this, let $\tilde{\nabla}$ be any affine connection on $M$ and let

$$
\tilde{\eta}(X)=\operatorname{tr}\left(Y \mapsto \tilde{\nabla}_{Y} X\right) .
$$

Then we obtain an affine connection $\nabla$ by setting

$$
\nabla_{X} Y=\Pi_{X} Y+\frac{1}{n+1} \tilde{\eta}(X) Y+\frac{1}{n+1} \tilde{\eta}(Y) X .
$$

Any other affine connection thus constructed is projectively equivalent to $\nabla$.

If we begin with an affine connection, construct $\Pi$ from it as in the first part of the proof, then use $\Pi$ to construct $\nabla$ as above, it is easily verified that $\nabla$ is projectively equivalent to the original affine connection. Therefore the correspondence between reduced complex projective connections and projective equivalence classes of affine connections is one-to-one. 
Corollary 4.4. Every complex manifold has a reduced complex projective connection.

It is not the case, however, that every complex manifold has a holomorphic projective connection. The Chern classes of a complex manifold which admits a holomorphic projective connection must satisfy certain relations (see [6], [7], [10]). From this one obtains examples of manifolds which do not admit a holomorphic projective connection. More examples are given in [8]. A complete characterization of the compact complex surfaces which admit a holomorphic projective connection was given by Kobayashi and Ochiai [10]; they went on to show that all surfaces which admit a holomorphic projective connection admit a flat holomorphic projective connection $[11]$.

\section{Principal Bundle Approach to Holomorphic Projective Connections}

Projective structures and connections on differentiable manifolds were described by E. Cartan in the language of principal bundles [1]. The relationships between Cartan's approach to projective connections on differentiable manifolds and that of H. Weyl and T.Y. Thomas have been studied by several authors including Kobayashi and Nagano [9].

In the study of projective structure on complex manifolds, the principal bundle approach was used by Ganesh [4]. Ganesh's approach to complex projective connections is similar to Gunning's approach and uses local coordinate representation of projective connections. In this section, we show that one may define a complex projective connection as a certain Cartan connection. The complex analog of a normal projective connection in the sense of Cartan then corresponds to a reduced complex projective connection in the sense of Section 4. The development of projective connections in the sense of Cartan on complex manifolds is formally very similar to the development on differentiable manifolds as given by Kobayashi and Nagano [9]. Here we only outline the main ideas and indicate how a complex projective Cartan connection is related to Definition 4.1.

Let $G^{2}(n)$ denote the group of 2-jets of locally invertible holomorphic mappings on a neighborhood of 0 in $\mathbf{C}^{n}$ which map 0 to itself. Let $P^{2}(M)$ be the $G^{2}(n)$ principal bundle of 2-jets of locally invertible holomorphic mappings from a neighborhood of 0 in $\mathbf{C}^{n}$ to $M$. The action of $G^{2}(n)$ on $P^{2}(M)$ is determined by composition of mappings.

Let $(U, z)$ be a local holomorphic coordinate neighborhood on $M$ and let $\zeta=$ $\left(\zeta^{1}, \ldots, \zeta^{n}\right)$ be standard complex Euclidean coordinates on $\mathbf{C}^{n}$. Then there is a natural coordinate system on $P^{2}(M)$ which we denote by $\left(s^{i}, s_{j}^{i}, s_{j k}^{i}\right)$. The representation of a 2 -jet of a mapping $f$ is given in these coordinates by

$$
s^{i}=f^{i}(0), \quad s_{j}^{i}=\frac{\partial f^{i}}{\partial \zeta^{j}}(0), \quad s_{j k}^{i}=\frac{\partial^{2} f^{i}}{\partial \zeta^{j} \partial \zeta^{k}}(0) .
$$

Here $f^{i}=z^{i} \circ f$. If $M=\mathbf{C}^{n}$ we obtain natural coordinates on $G^{2}(n)$ which we denote by $\left(t_{j}^{i}, t_{j k}^{i}\right)$.

The Schwarzian derivative of a mapping $f$ depends only on its 2-jet $\left(t_{j}^{i}, t_{j k}^{i}\right)$ :

$$
S(f)_{i j}^{k}=\tilde{t}_{r}^{i} t_{j k}^{r}-\frac{\delta_{j}^{i}}{n+1} \tilde{t}_{m}^{l} t_{l k}^{m}-\frac{\delta_{k}^{i}}{n+1} \tilde{t}_{m}^{l} t_{l j}^{m},
$$


where $\left(\tilde{t}_{j}^{i}\right)$ is the inverse matrix of $\left(t_{j}^{i}\right)$. We will write $S(t)$ for the Schwarzian derivative of the 2 -jet $\left(t_{j}^{i}, t_{j k}^{i}\right)$.

Let $H^{2}(n) \subset G^{2}(n)$ be the subgroup of 2-jets of complex projective linear mappings. In the natural coordinate system on $G^{2}(n)$, the group $H^{2}(n)$ is the subgroup of 2-jets which satisfy $S(t)=0$.

One can choose a coordinate system on $H^{2}(n)$ by writing a projective linear mapping in holomorphic Euclidean coordinates:

$$
\zeta \mapsto \frac{A_{j}^{i} \zeta^{j}+A_{0}^{i}}{A_{l}^{0} \zeta^{l}+A_{0}^{0}}
$$

If the mapping preserves the origin, then $A_{0}^{i}=0$. In a neighborhood of the identity mapping, we may divide through by $A_{0}^{0}$ and write the mapping as

$$
\zeta \mapsto \frac{a_{j}^{i} \zeta^{j}}{a_{l} \zeta^{l}+1},
$$

where $a_{j}^{i}=A_{j}^{i} / A_{0}^{0}$ and $a_{l}=A_{l}^{0} / A_{0}^{0}$. This gives a set of local holomorphic coordinates for $H^{2}(n)$ near the identity, namely $\left(a_{j}^{i}, a_{l}\right)$.

Now suppose that $M$ is a complex manifold with a complex projective structure. Let $\left\{\left(U_{\alpha}, z_{\alpha}\right)\right\}$ denote a maximal projective atlas. Ganesh [4] shows that there is an associated reduction of the structure group of $P^{2}(M)$ to $H^{2}(n)$. Let $P$ denote the associated $H^{2}(n)$ holomorphic subbundle of $P^{2}(M)$.

Let $\operatorname{Red}\left\{P^{2}(M), H^{2}(n)\right\}$ denote the reductions of the structure group of $P^{2}(M)$ to $H^{2}(n)$ modulo isomorphisms of the associated $H^{2}(n)$ principal subbundle. The relationship between $\operatorname{Red}\left\{P^{2}(M), H^{2}(n)\right\}$ and complex projective connections is given by the following result.

Proposition 5.1. The set $\operatorname{Red}\left\{P^{2}(M), H^{2}(n)\right\}$ is in one-to-one correspondence with the set of reduced holomorphic projective connections.

Proof. Ganesh proves that each reduction gives a reduced holomorphic projective connection $\Pi([4])$. To prove that the map is surjective, suppose that $\Pi$ is a reduced holomorphic projective connection. Ganesh shows that one obtains a reduction if there are 2-jets $\left(t_{\alpha j}^{i}, t_{\alpha j k}^{i}\right)$ such that

$$
\Pi_{\alpha j k}^{i}=S\left(t_{\alpha}\right) .
$$

Since $\Pi$ is symmetric and reduced, one may take $\left(t_{\alpha j}^{i}, t_{\alpha j k}^{i}\right)=\left(\delta_{j}^{i}, \Pi_{\alpha j k}^{i}\right)$.

We call a reduction flat if the corresponding reduced complex projective connection is flat. There is a one-to-one correspondence between the complex projective structures on $M$, the flat reductions of structure group of $P^{2}(M)$ to $H^{2}(n)$, and the flat reduced holomorphic projective connections on $M$.

Finally we wish to establish the relationship between the Cartan connections and the projective connections of Definition 4.1.

Let $\mathfrak{p l}(n, \mathbf{C})$ denote the Lie algebra of $P L(n, \mathbf{C})$. Suppose $P \subset P^{2}(M)$ is a holomorphic principal $H^{2}(n)$ bundle associated with a reduction (write $P \in$ $\operatorname{Red}\left\{P^{2}(M), H^{2}(n)\right\}$ to denote a reduction and associated bundle). A projective Cartan connection is a $\mathfrak{p l}(n, \mathbf{C})$-valued form of type $(1,0)$ on $P$ which satisfies the definition of a Cartan connection (see [9, p.218]). Following Kobayashi and Nagano [9] in the differentiable manifold case, we denote such a form by

$$
\omega=\left(\omega^{i}, \omega_{j}^{i}, \omega_{j}\right)
$$


corresponding to the standard decomposition of $\mathfrak{p l}(n, \mathbf{C})$. The structure equations of the connection form $\omega$ are

$$
\begin{aligned}
d \omega^{i} & =-\omega_{k}^{i} \wedge \omega^{k}+\Omega^{i}, \\
d \omega_{j}^{i} & =-\omega_{k}^{i} \wedge \omega_{j}^{k}-\omega^{i} \wedge \omega_{j}+\delta_{j}^{i} \omega_{k} \wedge \omega^{k}+\Omega_{j}^{i}, \\
d \omega_{j} & =-\omega_{k} \wedge \omega_{j}^{k}+\Omega_{j} .
\end{aligned}
$$

These equations define the forms $\Omega^{i}, \Omega_{j}^{i}$, $\Omega_{j}$, which may be written as

$$
\Omega^{i}=\frac{1}{2} K_{k l}^{i} \omega^{k} \wedge \omega^{l}, \quad \Omega_{j}^{i}=\frac{1}{2} K_{j k l}^{i} \omega^{k} \wedge \omega^{l}, \quad \Omega_{j}=\frac{1}{2} K_{j k l} \omega^{k} \wedge \omega^{l} .
$$

Let $\theta=\left(\theta^{i}, \theta_{j}^{i}\right)$ denote the canonical form on $P^{2}(M)$. The form $\theta$ is defined exactly as in the differentiable manifold case. In the complex case, $\theta$ is a holomorphic $(1,0)$ form with values in $\mathfrak{a}(n, \mathbf{C})$, where $\mathfrak{a}(n, \mathbf{C})$ is the Lie algebra of the affine group; $\mathfrak{a}(n, \mathbf{C})=\mathbf{C}^{n}+\mathfrak{g l}(n, \mathbf{C})$. Let $\bar{\theta}=\left(\bar{\theta}^{i}, \bar{\theta}_{j}^{i}\right)$ denote the restriction of $\theta$ to $P$. Because we assume $P$ is a holomorphic subbundle of $P^{2}(M), \bar{\theta}$ is a holomorphic $(1,0)$-form on $P$. As in the differentiable case, one may assign a unique Cartan connection to a holomorphic reduction $P \in \operatorname{Red}\left\{P^{2}(M), H^{2}(n)\right\}$. This is the analog of Theorem 9 of [9]:

Proposition 5.2. For each holomorphic reduction $P \in \operatorname{Red}\left\{P^{2}(M), H^{2}(n)\right\}$ there is a unique holomorphic projective Cartan connection $\omega=\left(\omega^{i}, \omega_{j}^{i}, \omega_{j}\right)$ such that

$$
\omega^{i}=\bar{\theta}^{i}, \quad \omega_{j}^{i}=\bar{\theta}_{j}^{i}, \quad d \omega^{i}=-\omega_{k}^{i} \wedge \omega^{k}, \quad \Omega_{i}^{i}=0, \quad K_{j i l}^{i}=0 .
$$

We call this Cartan connection the normal projective Cartan connection of the holomorphic reduction $P$. Finally we relate this connection to the previously defined projective connection.

Proposition 5.3. Let $P \in \operatorname{Red}\left\{P^{2}(M), H^{2}(n)\right\}$ be a holomorphic reduction. Let $(U, z)$ be a holomorphic coordinate neighborhood on $M$ with $\left.P\right|_{U} \simeq U \times H^{2}(n)$. Suppose that $\Pi$ is the reduced complex projective connection associated with $P$ with representation $\Pi_{j k}^{i}$ in the local coordinates. Let $\omega$ be the normal projective Cartan connection associated with $P$. Let $\left(z^{i}, a_{j}^{i}, a_{j}\right)$ denote local coordinates on $\left.P\right|_{U} \simeq$ $U \times H^{2}(n)$. Let $\left(b_{j}^{i}\right)$ be the inverse matrix of $\left(a_{j}^{i}\right)$. Then $\omega$ and $\Pi$ are related by the equations

$$
\begin{aligned}
\omega^{i} & =b_{l}^{i} d z^{l}, \\
\omega_{j}^{i} & =b_{m}^{i} d a_{j}^{m}+a_{j} \omega^{i}+a_{j}^{l} b_{k}^{i} \Pi_{m l}^{k} d z^{m}+\delta_{j}^{i} a_{k} \omega^{k}, \\
\omega_{j} & =d a_{j}-a_{k} \omega_{j}^{k}+a_{j} a_{k} \omega^{k}+a_{j}^{k} \Pi_{k l} d z^{l},
\end{aligned}
$$

where

$$
\Pi_{i j}=-\left(\frac{\partial \Pi_{i j}^{l}}{\partial z^{l}}-\Pi_{i l}^{m} \Pi_{j m}^{l}\right) .
$$

Proof. Adoption of the arguments used in [9] to the holomorphic case tells us that the form $\omega$ has the given expression in local coordinates for some choice of functions $\Pi_{m l}^{k}$ and $\Pi_{k l}$ in (2). Since the normal projective Cartan connection is unique, we need only show that with $\Pi_{m l}^{k}$ and $\Pi_{k l}$ as given - that is, taking $\Pi_{m l}^{k}$ to be the local representation of the reduced complex projective connection and $\Pi_{k l}$ as in (3) - the forms $\omega^{i}, \omega_{j}^{i}$, and $\omega_{j}$ satisfy the conditions of Proposition 5.2. 
We first verify $d \omega^{i}=-\omega_{k}^{i} \wedge \omega^{k}$. From (2) we have

$$
\omega_{k}^{i} \wedge \omega^{k}=\left(b_{m}^{i} d a_{k}^{m}+a_{k} \omega^{i}+a_{k}^{l} b_{j}^{i} \Pi_{m l}^{j} d z^{m}+\delta_{k}^{i} a_{l} \omega^{l}\right) \wedge \omega^{k} .
$$

Now using the symmetry of $\Pi\left(\Pi_{m l}^{j}=\Pi_{l m}^{j}\right)$ and the equations (2), we see that the term involving $\Pi$ vanishes and we obtain

$$
\omega_{k}^{i} \wedge \omega^{k}=b_{m}^{i} d a_{k}^{m} \wedge \omega^{k}=-d \omega^{i} .
$$

To check $\Omega_{i}^{i}=0$, again use (2) to obtain

$$
\begin{aligned}
\omega_{i}^{i} & =b_{m}^{i} d a_{i}^{m}+a_{i} \omega^{i}+a_{i}^{l} b_{k}^{i} \Pi_{m l}^{k} d z^{m}+n a_{k} \omega^{k} \\
& =b_{m}^{i} d a_{i}^{m}+(n+1) a_{k} \omega^{k} .
\end{aligned}
$$

Here we used the property of a reduced connection, $\Pi_{m k}^{k}=0$. The structure equations now easily give $\Omega_{i}^{i}=0$.

Verification of $K_{j i l}^{i}=0$ follows by similar calculation. In fact the expression (3) for $\Pi_{i j}$ is forced by this curvature condition and the expressions for $\omega^{i}$ and $\omega_{j}^{i}$. The calculation is simplified considerably by noting that since $K_{j i l}^{i}=0$ identically in the coordinates $\left(z^{i}, a_{j}^{i}, a_{j}\right)$, it must hold for the particular choice $\left(a_{j}^{i}, a_{j}\right)=\left(\delta_{j}^{i}, 0\right)$.

This proposition establishes the link between the Cartan connection approach to complex projective connections and the approach used by Gunning [6] and Ganesh [4].

\section{First Definition of Schwarzian Derivative}

Let $M$ and $N$ be complex manifolds of dimension $n>1$. We fix complex projective connections, denoted $\Pi^{M}$ and $\Pi^{N}$, on $M$ and $N$. Let $\phi: M \rightarrow N$ be locally biholomorphic. We wish to define the Schwarzian derivative of $\phi$ to be a section of $T^{h *} M \otimes T^{h *} M \otimes T^{h} M$ which measures the extent to which $\phi$ does not preserve the projective connection. Given the structure of a complex projective connection, the definition is simple:

Definition 6.1. The Schwarzian derivative of $\phi$ is the operator $\Sigma_{\phi}$ defined for $X, Y \in \mathfrak{X}^{h} M$ by

$$
\Sigma_{\phi}(X, Y)=\phi_{*}^{-1} \Pi_{\phi_{*} X}^{N} \phi_{*} Y-\Pi_{X}^{M} Y .
$$

Proposition 6.1. $\Sigma_{\phi}$ has the following properties.

1. $\Sigma_{\phi}$ is a symmetric tensor.

2. If $\Pi^{M}$ and $\Pi^{N}$ are holomorphic projective connections, then $\Sigma_{\phi}$ is a holomorphic tensor.

3. If $\Pi^{M}$ and $\Pi^{N}$ are both flat, then in any local projective coordinates on $M$ and $N, \Sigma_{\phi}$ is the classical Schwarzian defined in Section 3.

Proof. The symmetry of $\Sigma_{\phi}$ follows from property 5 in Definition 4.1 ; that $\Sigma_{\phi}$ is a tensor follows from properties $1-4$. It is obvious from the definition that if $\Pi^{M}$ and $\Pi^{N}$ are holomorphic, then $\Sigma_{\phi}$ is holomorphic.

If $\Pi^{M}$ and $\Pi^{N}$ are flat holomorphic projective connections, fix local projective coordinates $\left(x^{1}, \ldots, x^{n}\right)$ on $M$ and $\left(z^{1}, \ldots z^{n}\right)$ on $N, z^{i}=\phi^{i}(x)$. A direct computation yields

$$
\Sigma_{\phi}\left(\frac{\partial}{\partial x^{i}}, \frac{\partial}{\partial x^{j}}\right)=\frac{\partial z^{l}}{\partial x^{i}} \frac{\partial x^{m}}{\partial x^{j}} \Pi_{l m}^{N} \frac{\partial x^{k}}{\partial z^{q}} \frac{\partial}{\partial x^{k}}-\Pi^{M_{i j}^{k}} \frac{\partial}{\partial x^{k}} .+S(\phi)\left(\frac{\partial}{\partial x^{i}}, \frac{\partial}{\partial x^{j}}\right) .
$$


Since $\Pi^{N_{l m}^{q}}$ and $\Pi^{M^{k}}{ }_{i j}$ vanish, we see that in these coordinates, $\Sigma_{\phi}=S(\phi)$.

\section{The Associated Line Bundle}

In [17], Thomas showed that associated to a differentiable manifold $V$ with differentiable projective connection, there is a manifold ${ }^{*} V$ of one higher dimension with an affine connection which is related to the projective connection on $V$. The projective geometry of $V$ is encoded in the affine geometry of ${ }^{*} V$. In modern language, one would say that ${ }^{*} V$ is a line bundle over $V$. The prototypical example is $V=\mathbf{R} P^{n},{ }^{*} V=\mathbf{R}^{n+1}-\{0\},{ }^{*} V \rightarrow V$ the usual quotient map.

We next carry out a similar construction for a complex manifold with complex projective connection and show how this idea can be used to define the Schwarzian derivative in an illuminating way.

Let $M$ be a complex manifold of dimension $n>1$. We must make the assumption that there is a holomorphic line bundle $\hat{M} \stackrel{\pi}{\rightarrow} M$ whose $(n+1)$ st tensor power is the canonical line bundle $\kappa=\wedge^{n} T^{h *} M$.

If $M$ has a complex projective structure represented by an atlas $\left\{\left(U_{\alpha}, x_{\alpha}\right)\right\}$ with coordinate changes

$$
x_{\beta}^{i}=f_{\alpha \beta}^{i}\left(x_{\alpha}\right)=\frac{a_{j}^{i} x_{\alpha}^{j}+b^{i}}{c_{j} x_{\alpha}^{j}+d},
$$

one may normalize so that $\operatorname{det}\left(\begin{array}{ll}a_{j}^{i} & b^{i} \\ c_{j} & d\end{array}\right)=1$ by multiplying the numerator and denominator by an appropriate constant. Then the Jacobian determinant of $f_{\alpha \beta}$ is $g_{\alpha \beta}=\left(c_{j} x_{\alpha}^{j}+d\right)^{-(n+1)}$ and $\left\{g_{\alpha \beta}^{-1}\right\}$ is a set of transition functions for $\kappa$. Choose $h_{\alpha \beta}$ to be an $(n+1)$ st root of $g_{\alpha \beta}^{-1}$. One could, in particular, choose $h_{\alpha \beta}=c_{j} x_{\alpha}^{j}+d$. Then $\left(h_{\alpha \beta} h_{\beta \gamma} h_{\gamma \alpha}\right)^{n+1}=\left(g_{\alpha \beta} g_{\beta \gamma} g_{\gamma \alpha}\right)^{-1}=1$, so $H_{\alpha \beta \gamma}=h_{\alpha \beta} h_{\beta \gamma} h_{\gamma \alpha}$ is an $(n+1)$ st root of unity. Observe that $\left\{H_{\alpha \beta \gamma}\right\}$ is a cocycle with values in $\mathbf{Z} / n+1$. If the cohomology class of $\left\{H_{\alpha \beta \gamma}\right\}$ in $H^{2}(M, \mathbf{Z} / n+1)$ vanishes, then there is a cochain $\left\{F_{\alpha \beta}\right\}$ with values in $\mathbf{Z} / n+1$ such that $F_{\alpha \beta} F_{\beta \gamma} F_{\gamma \alpha}=H_{\alpha \beta \gamma}$. Let $\tilde{h}_{\alpha \beta}=h_{\alpha \beta} F_{\alpha \beta}^{-1}$. Then $\tilde{h}_{\alpha \beta}$ is an $(n+1)$ st root of $g_{\alpha \beta}^{-1}$ and $\tilde{h}_{\alpha \beta} \tilde{h}_{\beta \gamma} \tilde{h}_{\gamma \alpha}=1$. Therefore $\left\{\tilde{h}_{\alpha \beta}\right\}$ is a set of transition functions for the required bundle $\hat{M}$.

The cohomological condition for the existence of $\hat{M}$ is further discussed in [16]; it is always satisfied when $n=1$. For $M=\mathbf{C} P^{n}$, we may take $\hat{M} \simeq \mathcal{O}(-1)$, the tautological bundle on complex projective space.

Now return to a complex manifold $M$ supporting such a bundle $\hat{M}$, but not necessarily having a complex projective structure. Let $\left(x_{\alpha}^{1}, \ldots, x_{\alpha}^{n}\right)$ and $\left(x_{\beta}^{1}, \ldots, x_{\beta}^{n}\right)$ be local holomorphic coordinates on $M$. Let

$$
\Delta_{\alpha \beta}=\left(\operatorname{det} \frac{\partial x_{\beta}}{\partial x_{\alpha}}\right)^{\frac{-1}{n+1}} .
$$

The $(n+1)$ st roots are chosen so that $\Delta_{\alpha \beta}$ are the transition functions of the bundle $\hat{M}$. Then local coordinates on $\hat{M}$ are given by $\left(x_{\alpha}^{0}, x_{\alpha}^{\prime}\right)=\left(x_{\alpha}^{0}, x_{\alpha}^{1}, \ldots, x_{\alpha}^{n}\right)$ and $\left(x_{\beta}^{0}, x_{\beta}^{\prime}\right)=\left(x_{\beta}^{0}, x_{\beta}^{1}, \ldots, x_{\beta}^{n}\right)$ with coordinate transformations

$$
\begin{aligned}
& x_{\beta}^{i}=f_{\alpha \beta}^{i}\left(x_{\alpha}^{\prime}\right) \quad \text { for } i>0, \\
& x_{\beta}^{0}=\Delta_{\alpha \beta}\left(x_{\alpha}^{\prime}\right) x_{\alpha}^{0} .
\end{aligned}
$$


Let $\sigma \subset \hat{M}$ denote the zero section. On $\hat{M}-\sigma$, we introduce "homogeneous coordinates":

$$
\begin{aligned}
& X_{\alpha}^{0}=x_{\alpha}^{0}, \\
& X_{\alpha}^{i}=x_{\alpha}^{0} x_{\alpha}^{i} \quad \text { for } i>0 .
\end{aligned}
$$

The coordinate transformations are

$$
\begin{aligned}
& X_{\beta}^{0}=X_{\alpha}^{0} \Delta_{\alpha \beta}\left(\frac{X_{\alpha}^{1}}{X_{\alpha}^{0}}, \ldots, \frac{X_{\alpha}^{n}}{X_{\alpha}^{0}}\right), \\
& X_{\beta}^{i}=X_{\alpha}^{0} \Delta_{\alpha \beta}\left(\frac{X_{\alpha}^{1}}{X_{\alpha}^{0}}, \ldots, \frac{X_{\alpha}^{n}}{X_{\alpha}^{0}}\right) f_{\alpha \beta}^{i}\left(\frac{X_{\alpha}^{1}}{X_{\alpha}^{0}}, \ldots, \frac{X_{\alpha}^{n}}{X_{\alpha}^{0}}\right) .
\end{aligned}
$$

Proposition 7.1. If $M$ has a complex projective atlas, then $\hat{M}$ has a complex affine atlas.

Proof. On $M$, fix a covering of coordinate charts such that the coordinate transformations are complex projective linear:

$$
x_{\beta}^{i}=\frac{a_{j}^{i} x_{\alpha}^{j}+b^{i}}{c_{j} x_{\alpha}^{j}+d} .
$$

We may normalize so that

$$
\operatorname{det}\left(\begin{array}{cc}
a_{j}^{i} & b^{i} \\
c_{j} & d
\end{array}\right)=1
$$

After multiplying the matrix by an $(n+1)$ st root of unity, we may further assume that $\Delta_{\alpha \beta}=c_{j} x_{\alpha}^{j}+d$; the existence of $\hat{M}$ means that an $(n+1)$ st root can be so chosen. The homogeneous coordinate transformations are then

$$
\begin{aligned}
& X_{\beta}^{0}=c_{j} X_{\alpha}^{j}+d X_{\alpha}^{0}, \\
& X_{\beta}^{i}=a_{j}^{i} X_{\alpha}^{j}+b^{i} X_{\alpha}^{0} \text { for } i>0,
\end{aligned}
$$

which are affine (in fact linear) transformations.

It is clear from the proof of Proposition 7.1 that a converse of sorts is true: if $\hat{M}$ has a covering by homogeneous coordinates with linear coordinate changes, then $M$ has a complex projective structure.

We next define on $\hat{M}-\sigma$ a global $(n+1,0)$-form which will be needed in the next section.

Proposition 7.2. Define an $(n+1,0)$-form $\nu_{\alpha}=d X_{\alpha}^{0} \wedge \cdots \wedge d X_{\alpha}^{n}$ on $\pi^{-1} U_{\alpha}$. Then on $\pi^{-1} U_{\alpha} \cap \pi^{-1} U_{\beta}, \nu_{\alpha}=\nu_{\beta}$.

Proof. A direct computation shows that

$$
\operatorname{det}\left(\frac{\partial x_{\beta}^{J}}{\partial x_{\alpha}^{I}}\right) \equiv 1 .
$$

The global $(n+1,0)$-form thus defined will be denoted $\nu$ or $\nu_{\hat{M}}$. Notice that $d V=\nu \wedge \bar{\nu}$ is a volume form on $\hat{M}$.

The vector field on $\hat{M}$ defined by $\xi=x_{\alpha}^{0} \partial / \partial x_{\alpha}^{0}$ is a global holomorphic vector field. It will be used in Theorem 7.3. The vector field $\xi$ is vertical: $\pi_{*} \xi=0$.

Fix a reduced complex projective connection $\Pi$ on $M$. We use the projective connection $\Pi$ to introduce an affine connection on $\hat{M}$. As mentioned in Section 2, lower case Roman indices will take on values from 1 to $n$, upper case from 0 to $n$. 
Theorem 7.3. There is a unique affine connection $\nabla$ on $\hat{M}-\sigma$ satisfying

1. For $\left(x_{\alpha}^{1}, \ldots, x_{\alpha}^{n}\right)$ local coordinates on $M$, and $\left(x_{\alpha}^{0}, x_{\alpha}^{\prime}\right)$ the corresponding local coordinates on $\hat{M}, \pi_{*} \nabla_{\partial / \partial x_{\alpha}^{i}} \frac{\partial}{\partial x_{\alpha}^{j}}$ is defined and equals $\Pi_{\partial / \partial x_{\alpha}^{i}} \frac{\partial}{\partial x_{\alpha}^{j}}$.

2. $\nabla_{X} \xi=X$ for all $X \in T^{h} \hat{M}$

3. $R(X, \xi) Y=0$ for all $X, Y \in T^{h} \hat{M}$

4. $\operatorname{Ric} \nabla(X, Y)=0$ for all $X, Y \in T^{h} \hat{M}$

Proof. Let $\Gamma_{\alpha_{I J}}^{K}$ be the Christoffel symbols of the desired connection on $\hat{M}-\sigma$ in terms of the $\left(x_{\alpha}^{0}, \ldots, x_{\alpha}^{n}\right)$ coordinates. We show that $\Gamma_{\alpha I J}^{K}$ are uniquely determined by conditions 1-4 and that they transform correctly under a change of coordinates.

For the first part of the computation, we will work with only one coordinate chart, so we drop the subscript $\alpha$. From condition 1, if we write

$$
\nabla_{\frac{\partial}{\partial x^{i}}} \frac{\partial}{\partial x^{j}}=\Gamma_{i j}^{0} \frac{\partial}{\partial x^{0}}+\Gamma_{i j}^{k} \frac{\partial}{\partial x^{k}}
$$

then

so $\Gamma_{i j}^{k}=\Pi_{\alpha i j}^{k}$.

$$
\pi_{*} \nabla_{\frac{\partial}{\partial x^{i}}} \frac{\partial}{\partial x^{j}}=\Gamma_{i j}^{k} \frac{\partial}{\partial x^{k}},
$$

Let $X=\frac{\partial}{\partial x^{2}}$. Then condition 2 says

$$
\frac{\partial}{\partial x^{i}}=\nabla_{\frac{\partial}{\partial x^{i}}}\left(x^{0} \frac{\partial}{\partial x^{0}}\right)=x^{0} \Gamma_{i 0}^{0} \frac{\partial}{\partial x^{0}}+x^{0} \Gamma_{i 0}^{k} \frac{\partial}{\partial x^{k}},
$$

so $\Gamma_{i 0}^{0}=0$ and $\Gamma_{i 0}^{k}=\delta_{i}^{k} / x^{0}$. By symmetry, $\Gamma_{0 i}^{0}=0$ and $\Gamma_{0 i}^{k}=\delta_{i}^{k} / x^{0}$. Next let $X=\frac{\partial}{\partial x^{0}}$. Condition 2 then implies

$$
\frac{\partial}{\partial x^{0}}=\nabla_{\frac{\partial}{\partial x^{0}}}\left(x^{0} \frac{\partial}{\partial x^{0}}\right)=\frac{\partial}{\partial x^{0}}+x^{0} \Gamma_{00}^{0} \frac{\partial}{\partial x^{0}}+x^{0} \Gamma_{00}^{k} \frac{\partial}{\partial x^{k}},
$$

so $\Gamma_{00}^{0}=0$ and $\Gamma_{00}^{k}=0$.

It remains to compute $\Gamma_{i j}^{0}$. First observe that

$$
\nabla_{\xi} \frac{\partial}{\partial x^{I}}=\nabla_{\frac{\partial}{\partial x^{I}}} \xi+\left[\xi, \frac{\partial}{\partial x^{I}}\right]=\frac{\partial}{\partial x^{I}}-\delta_{I}^{0} \frac{\partial}{\partial x^{0}}
$$

Then for $X=\frac{\partial}{\partial x^{i}}, Y=\frac{\partial}{\partial x^{j}}$, condition 3 says

$$
\begin{aligned}
0 & =R\left(\frac{\partial}{\partial x^{i}}, \xi\right) \frac{\partial}{\partial x^{j}} \\
& =\nabla_{\frac{\partial}{\partial x^{i}}} \nabla_{\xi} \frac{\partial}{\partial x^{j}}-\nabla_{\xi} \nabla_{\frac{\partial}{\partial x^{i}}} \frac{\partial}{\partial x^{j}}-\nabla_{\left[\frac{\partial}{\partial x^{i}}, \xi\right]} \frac{\partial}{\partial x^{j}} \\
& =\nabla_{\frac{\partial}{\partial x^{i}}} \frac{\partial}{\partial x^{j}}-\nabla_{\xi}\left(\Gamma_{i j}^{0} \frac{\partial}{\partial x^{0}}+\Gamma_{i j}^{k} \frac{\partial}{\partial x^{k}}\right)-\nabla_{0} \frac{\partial}{\partial x^{j}} \\
& =\Gamma_{i j}^{0} \frac{\partial}{\partial x^{0}}+\Gamma_{i j}^{k} \frac{\partial}{\partial x^{k}}-\xi\left(\Gamma_{i j}^{0}\right) \frac{\partial}{\partial x^{0}}-\xi\left(\Gamma_{i j}^{k}\right) \frac{\partial}{\partial x^{k}}-\Gamma_{i j}^{0} \cdot 0-\Gamma_{i j}^{k} \frac{\partial}{\partial x^{k}} \\
& =\left(\Gamma_{i j}^{0}-\xi\left(\Gamma_{i j}^{0}\right)\right) \frac{\partial}{\partial x^{0}}-\xi\left(\Gamma_{i j}^{k}\right) \frac{\partial}{\partial x^{k}} .
\end{aligned}
$$

Therefore $\xi\left(\Gamma_{i j}^{k}\right)=0$, so $\Gamma_{i j}^{k}$ must be independent of $x^{0}$. This is consistent with condition (1). Furthermore, $\xi\left(\Gamma_{i j}^{0}\right)=\Gamma_{i j}^{0}$, so

$$
x^{0} \frac{\partial \Gamma_{i j}^{0}}{\partial x^{0}}=\Gamma_{i j}^{0} .
$$


The solution of this differential equation is $\Gamma_{i j}^{0}=x^{0} \phi_{i j}\left(x^{\prime}\right)$, for any function $\phi_{i j}$.

To determine the functions $\phi_{i j}$, we use condition 4 . Let

$$
R_{i j}=\operatorname{Ric} \nabla\left(\frac{\partial}{\partial x^{i}}, \frac{\partial}{\partial x^{j}}\right) .
$$

We compute:

$$
\begin{aligned}
R_{i j}= & \Gamma_{K i}^{L} \Gamma_{j L}^{K}-\Gamma_{i j}^{L} \Gamma_{K L}^{K}+\frac{\partial \Gamma_{K i}^{K}}{\partial x^{j}}-\frac{\partial \Gamma_{i j}^{K}}{\partial x^{K}} \\
= & \Gamma_{0 i}^{0} \Gamma_{j 0}^{0}+\Gamma_{0 i}^{l} \Gamma_{j l}^{0}+\Gamma_{k i}^{0} \Gamma_{j 0}^{k}+\Gamma_{k i}^{l} \Gamma_{j l}^{k}-\Gamma_{i j}^{0} \Gamma_{00}^{0}-\Gamma_{i j}^{l} \Gamma_{0 l}^{0}-\Gamma_{i j}^{0} \Gamma_{k 0}^{k}-\Gamma_{i j}^{l} \Gamma_{k l}^{k} \\
& +\frac{\partial \Gamma_{0 i}^{0}}{\partial x^{j}}+\frac{\partial \Gamma_{k i}^{k}}{\partial x^{j}}-\frac{\partial \Gamma_{i j}^{0}}{\partial x^{0}}-\frac{\partial \Gamma_{i j}^{k}}{\partial x^{k}} \\
= & \frac{\delta_{i}^{l}}{x^{0}} \cdot x^{0} \phi_{j l}+x^{0} \phi_{k i} \frac{\delta_{j}^{k}}{x^{0}}+\Pi_{\alpha k i}^{l} \Pi_{\alpha j l}^{k}-x^{0} \phi_{i j} \frac{n}{x^{0}}-\frac{\partial}{\partial x^{0}}\left(x^{0} \phi_{i j}\right)-\frac{\partial \Pi_{\alpha i j}^{k}}{\partial x^{k}} \\
= & -(n-1) \phi_{i j}+B_{\alpha i j},
\end{aligned}
$$

where

$$
B_{\alpha i j}=\Pi_{\alpha k i}^{l} \Pi_{\alpha j l}^{k}-\frac{\partial \Pi_{\alpha i j}^{k}}{\partial x^{k}} .
$$

Then condition 4 implies that $\phi_{i j}=\frac{1}{n-1} B_{\alpha i j}$. A direct computation shows that under a change of coordinates $x_{\beta}=f_{\alpha \beta}\left(x_{\alpha}\right), B_{\alpha}$ transforms according to the law

$$
B_{\alpha i j}=\frac{\partial x_{\beta}^{p}}{\partial x_{\alpha}^{i}} \frac{\partial x_{\beta}^{q}}{\partial x_{\alpha}^{j}} B_{\beta p q}+\frac{n-1}{n+1}\left(\Pi_{\alpha i j}^{k}-S_{i j}^{k}\right) \frac{\partial}{\partial x_{\alpha}^{k}} \log J_{f_{\alpha \beta}}+S_{k i}^{l} S_{j l}^{k}-\frac{\partial}{\partial x_{\alpha}^{k}}\left(S_{i j}^{k}\right) .
$$

In (8), $S$ stands for the Schwarzian derivative $S\left(f_{\alpha \beta}\right)$. To sum up, we have found that conditions $1-4$ imply

$$
\begin{aligned}
\Gamma_{i j}^{k}=\Pi_{\alpha i j}^{k}, & \Gamma_{0 i}^{k}=\Gamma_{i 0}^{k}=\frac{\delta_{i}^{k}}{x^{0}}, \\
\Gamma_{i j}^{0}=\frac{x^{0}}{n-1} B_{\alpha i j}, & \Gamma_{i 0}^{0}=\Gamma_{0 i}^{0}=\Gamma_{00}^{0}=\Gamma_{00}^{k}=0 .
\end{aligned}
$$

It is easily seen, by direct computation, that for a connection with these Christoffel symbols, conditions 3 and 4 are satisfied for all vectors in $T^{h} \hat{M}$.

We now resume writing the subscripts $\alpha$ and $\beta$. It remains to verify that

$$
\Gamma_{\alpha I J}^{K}=\frac{\partial x_{\beta}^{L}}{\partial x_{\alpha}^{I}} \frac{\partial x_{\beta}^{P}}{\partial x_{\alpha}^{J}} \Gamma_{\beta}^{Q} \underset{L P}{Q} \frac{\partial x_{\alpha}^{K}}{\partial x_{\beta}^{Q}}+\frac{\partial^{2} x_{\beta}^{L}}{\partial x_{\alpha}^{I} \partial x_{\alpha}^{J}} \frac{\partial x_{\alpha}^{K}}{\partial x_{\beta}^{L}} .
$$

Observe that

$$
\frac{\partial x_{\beta}^{0}}{\partial x_{\alpha}^{0}}=\Delta_{\alpha \beta}, \quad \frac{\partial x_{\beta}^{0}}{\partial x_{\alpha}^{i}}=\frac{\partial \Delta_{\alpha \beta}}{\partial x_{\alpha}^{i}} x_{\alpha}^{0}, \quad \frac{\partial x_{\beta}^{i}}{\partial x_{\alpha}^{0}}=0 .
$$

For $I \neq 0, J \neq 0, K \neq 0$, equation (9) reduces to the transformation law (1) for $\Pi$. For $I=J=0$, and $K \neq 0$, and for $I=0$ or $J=0, K \neq 0$, all terms in equation (9) vanish identically. For $I \neq 0, J=0, K=0$ (resp. $I=0, J \neq 0, K=0)$, both sides of equation (9) reduce to $\partial / \partial x_{\alpha}^{I}\left(\log \Delta_{\alpha \beta}\right)\left(\right.$ resp. $\left.\partial / \partial x_{\alpha}^{J}\left(\log \Delta_{\alpha \beta}\right)\right)$. For $I \neq 0$, $J \neq 0, K=0$, equation (9) can be verified by using the transformation law (8) for $B_{\alpha i j}$. 
Observe that $B_{\alpha i j}$ is the same as $\Pi_{i j}$ defined in Proposition 5.3. Also note that if $\Pi$ is a holomorphic projective connection, then $\nabla$ is a holomorphic affine connection. If $\Pi$ is in addition flat and projective coordinates are chosen so that

$$
\Pi_{\frac{\partial}{\partial x_{\alpha}^{i}}} \frac{\partial}{\partial x_{\alpha}^{j}}=0
$$

for all $\alpha$, then $\Gamma_{\alpha 0 i}^{k}=\Gamma_{\alpha i 0}^{k}=\delta_{i}^{k} / x^{0}$ and all other $\Gamma_{\alpha I J}^{K}$ are identically zero. If one uses $C_{\alpha I J}^{K}$ to denote the Christoffel symbols in terms of the homogeneous coordinates $\left(X_{\alpha}^{0}, \ldots, X_{\alpha}^{n}\right)$, then it is easily verified that $C_{\alpha I J}^{K} \equiv 0$, so $\nabla$ is a flat connection.

\section{Second Definition of Schwarzian Derivative}

The fact that the projective geometry of $M$ is reflected in the affine geometry of $\hat{M}$ can be used to define the Schwarzian derivative in another way. Let $M$ and $N$ be two complex manifolds of the same dimension $n>1$. Suppose that line bundles $\hat{M}$ and $\hat{N}$ exist, and let $\nu_{\hat{M}}$ and $\nu_{\hat{N}}$ be the $(n+1,0)$-forms on $\hat{M}-\sigma$ and on $\hat{N}-\sigma$ defined in the previous section. Let $\phi: M \rightarrow N$ be locally biholomorphic.

In [13], locally biholomorphic maps from a subset $U$ of $\mathbf{C}^{n}$ to $\mathbf{C} P^{n}$ were considered. The Schwarzian was defined by using a canonical lift $\tilde{\phi}: U \rightarrow \mathbf{C}^{n+1}-\{0\}$ so that $\pi \circ \tilde{\phi}=\phi$. We will generalize this idea.

To define a canonical lift of $\phi$, no projective connection is needed:

Proposition 8.1. Given $M, N, \hat{M}, \hat{N}$ and $\phi$ as above, there is a holomorphic bundle map $\hat{\phi}: \hat{M} \rightarrow \hat{N}$ such that $\hat{\phi}^{*} \nu_{\hat{N}}=\nu_{\hat{M}}$. The map $\hat{\phi}$ is unique up to the choice of an $(n+1)$ st root of unity.

Proof. Let $x_{\alpha}^{\prime}=\left(x_{\alpha}^{1}, \ldots x_{\alpha}^{n}\right)$ be local coordinates on $U_{\alpha} \subset M, x_{\alpha}=\left(x_{\alpha}^{0}, x_{\alpha}^{\prime}\right)$ the corresponding local coordinates on $\pi^{-1} U_{\alpha} \subset \hat{M},\left(X_{\alpha}^{0}, \ldots X_{\alpha}^{n}\right)$ the corresponding homogeneous coordinates off the zero section of $\hat{M}$. Let $z^{\prime},\left(z^{0}, z^{\prime}\right)$, and $\left(Z^{0}, Z^{1}, \ldots, Z^{n}\right)$ denote the analogous coordinates for some coordinate patch in $\mathrm{N}$ which intersects $\phi\left(U_{\alpha}\right)$.

Let

$$
\left(z^{0}, z^{1}, \ldots, z^{n}\right)=\left(\hat{\phi}^{0}\left(x_{\alpha}\right), \hat{\phi}^{1}\left(x_{\alpha}\right), \ldots \hat{\phi}^{n}\left(x_{\alpha}\right)\right)
$$

and

$$
\left(z^{1}, \ldots, z^{n}\right)=\left(\phi^{1}\left(x_{\alpha}^{\prime}\right), \ldots, \phi^{n}\left(x_{\alpha}^{\prime}\right)\right)
$$

Because $\hat{\phi}$ is to be a bundle map, $\hat{\phi}^{i}\left(x_{\alpha}\right)=\phi^{i}\left(x_{\alpha}^{\prime}\right)$ for $i=1, \ldots, n$. Furthermore, $\hat{\phi}^{0}$ must be complex linear in $x_{\alpha}^{0}$, i.e. $\hat{\phi}^{0}\left(x_{\alpha}^{0}, x_{\alpha}^{\prime}\right)=x_{\alpha}^{0} \hat{\phi}_{\alpha}^{0}\left(1, x_{\alpha}^{\prime}\right)$. To determine $\hat{\phi}_{\alpha}^{0}\left(1, x_{\alpha}^{\prime}\right)$, observe that $\hat{\phi}$ preserves $\nu$ if and only if the determinant of the Jacobian matrix $\left(\partial Z^{I} / \partial X_{\alpha}^{J}\right)$ is identically equal to 1 . A direct computation yields

$$
\operatorname{det}\left(\frac{\partial Z^{I}}{\partial X_{\alpha}^{J}}\right)=\frac{\left(z^{0}\right)^{n}}{\left(x_{\alpha}^{0}\right)^{n}} \frac{\partial z^{0}}{\partial x_{\alpha}^{0}} \operatorname{det}\left(\frac{\partial z^{i}}{\partial x_{\alpha}^{j}}\right)=\left(\hat{\phi}_{\alpha}^{0}\left(1, x_{\alpha}^{\prime}\right)\right)^{n+1} \operatorname{det}\left(\frac{\partial z^{i}}{\partial x_{\alpha}^{j}}\right)_{i, j=1, \ldots, n} .
$$

Therefore $\hat{\phi}_{\alpha}^{0}\left(1, x_{\alpha}^{\prime}\right)$ is an $(n+1)$ st root of $\operatorname{det}\left(\partial z^{i} / \partial x_{\alpha}^{j}\right)$.

If we change coordinates on $\hat{M}$ to $\left(x_{\beta}^{0}, \ldots x_{\beta}^{n}\right)$, then, from (4), $x_{\beta}^{0}=\Delta_{\alpha \beta}\left(x_{\alpha}^{\prime}\right) x_{\alpha}^{0}$. Then $\hat{\phi}_{\beta}^{0}\left(1, x_{\beta}^{\prime}\right)$ is an $(n+1)$ st root of

$$
\operatorname{det}\left(\partial z^{i} / \partial x_{\beta}^{j}\right)=\operatorname{det}\left(\partial z^{i} / \partial x_{\alpha}^{j}\right) \operatorname{det}\left(\partial x_{\beta}^{i} / \partial x_{\alpha}^{j}\right)^{-1} .
$$


We may compute $\hat{\phi}_{\beta}^{0}\left(1, x_{\beta}^{\prime}\right)$ as follows:

$$
x_{\alpha}^{0} \hat{\phi}_{\alpha}^{0}\left(1, x_{\alpha}^{\prime}\right)=\hat{\phi}^{0}\left(x_{\alpha}^{0}, x_{\alpha}^{\prime}\right)=\hat{\phi}^{0}\left(x_{\beta}^{0}, x_{\beta}^{\prime}\right)=x_{\beta}^{0} \hat{\phi}_{\beta}^{0}\left(1, x_{\beta}^{\prime}\right)=x_{\alpha}^{0} \Delta_{\alpha \beta} \hat{\phi}_{\beta}^{0}\left(1, x_{\beta}^{\prime}\right) .
$$

Therefore $\hat{\phi}_{\beta}^{0}\left(1, x_{\beta}^{\prime}\right)=\Delta_{\alpha \beta}^{-1} \hat{\phi}_{\alpha}^{0}\left(1, x_{\alpha}^{\prime}\right)$.

From this we see that once $\hat{\phi}_{\alpha}^{0}\left(1, x_{\alpha}^{\prime}\right)$ has been chosen, $\hat{\phi}_{\beta}^{0}\left(1, x_{\beta}^{\prime}\right)$ is determined for all $U_{\beta}$ intersecting $U_{\alpha}$, and thus for all $U_{\gamma}$. Therefore $\hat{\phi}$ exists and is unique up to the choice of an $(n+1)$ st root of unity.

Now let $\Pi^{M}$ and $\Pi^{N}$ be reduced complex projective connections on $M$ and $N$, $\nabla^{\hat{M}}$ and $\nabla^{\hat{N}}$ the affine connections on $\hat{M}$ and $\hat{N}$ guaranteed by Theorem 7.3. Roughly speaking, the Schwarzian of $\phi$ should measure the extent to which $\hat{\phi}^{*} \nabla^{\hat{N}}$ differs from $\nabla^{\hat{M}}$.

Definition 8.1. For $X \in T^{h} \hat{M}, Y$ a local vector field with values in $T^{h} \hat{M}$, let

$$
\hat{\mathcal{D}}(X, Y)=\hat{\phi}_{*}^{-1} \nabla_{\hat{\phi}_{*}}^{\hat{N}} \hat{\phi}_{*} Y-\nabla_{X}^{\hat{M}} Y \text {. }
$$

Because it is a difference of two affine connections, $\hat{\mathcal{D}}$ is a tensor; more specifically, it is a symmetric section of $T^{h *} \hat{M} \otimes T^{h *} \hat{M}$.

Proposition 8.2. If $X=\xi_{M}$ or $Y=\xi_{M}$, then $\hat{\mathcal{D}}(X, Y)=0$.

Proof. First observe that $\hat{\phi}_{*} \xi_{M}=\xi_{N}$ by the construction of $\hat{\phi}$. Therefore, using condition 2 of Theorem 7.3 ,

$$
\hat{\mathcal{D}}\left(X, \xi_{M}\right)=\hat{\phi}_{*}^{-1} \nabla_{\hat{\phi}_{*}}^{\hat{N}} \xi_{N}-\nabla_{X}^{\hat{M}} \xi_{M}=\hat{\phi}_{*}^{-1} \hat{\phi}_{*} X-X=0
$$

Because $\hat{\mathcal{D}}$ is symmetric, the proof is complete.

We now compute locally. Let $U_{\alpha}$ be a coordinate patch in $M$, small enough so that $\left.\phi\right|_{U_{\alpha}}$ is biholomorphic and so that $\phi\left(U_{\alpha}\right)$ is contained in a coordinate patch $V_{\gamma} \subset N$. As before, let $\left(x_{\alpha}^{1}, \ldots x_{\alpha}^{n}\right)$ be coordinates on $U_{\alpha},\left(z_{\gamma}^{1}, \ldots z_{\gamma}^{n}\right)$ on $V_{\gamma}$. Since $\phi$ is locally biholomorphic, $\left(\phi\left(x_{\alpha}^{1}\right), \ldots, \phi\left(x_{\alpha}^{n}\right)\right)$ are also local coordinates on $V_{\gamma} \cap \phi\left(U_{\alpha}\right)$, and we will use $\Pi_{\alpha}^{N}$ and $B_{\alpha}^{N}$ to denote the complex projective connection and $B^{N}$ (defined by equation (7)) written in these coordinates.

\section{Proposition 8.3.}

$$
\hat{\mathcal{D}}\left(\frac{\partial}{\partial x_{\alpha}^{i}}, \frac{\partial}{\partial x_{\alpha}^{j}}\right)=\frac{1}{n-1}\left[B_{\alpha i j}^{N}-B_{\alpha i j}^{M}\right] \xi_{M}+\left[\Pi_{\alpha i j}^{N^{k}}-\Pi_{\alpha i j}^{M^{k}}\right] \frac{\partial}{\partial x_{\alpha}^{k}} .
$$


Proof. Let $J$ denote the Jacobian determinant of $z_{\gamma}=\phi\left(x_{\alpha}\right)$. Recall that $Z_{\gamma}^{0}=$ $J^{\frac{-1}{n+1}} x_{\alpha}^{0}$ and that $z_{\gamma}^{i}$ is independent of $x_{\alpha}^{0}$ for $i 0$. A direct computation yields

$$
\begin{aligned}
\hat{\phi}_{*}^{-1} \nabla_{\hat{\phi}_{*} \frac{\partial}{\partial x_{\alpha}^{i}}} \hat{\phi}_{*} \frac{\partial}{\partial x_{\alpha}^{j}} & {\left[\frac{\partial^{2} \log J^{\frac{-1}{n+1}}}{\partial x_{\alpha}^{i} \partial x_{\alpha}^{j}}-\frac{\partial \log J^{\frac{-1}{n+1}}}{\partial x_{\alpha}^{i}} \frac{\partial \log J^{\frac{-1}{n+1}}}{\partial x_{\alpha}^{j}}\right.} \\
& -\frac{\partial^{2} x_{\gamma}^{l}}{\partial x_{\alpha}^{i} \partial x_{\alpha}^{j}} \frac{\partial x_{\alpha}^{k}}{\partial z_{\gamma}^{l}} \frac{\partial \log J^{\frac{-1}{n+1}}}{\partial x_{\alpha}^{k}}+\frac{\partial z_{\gamma}^{k}}{\partial x_{\alpha}^{i}} \frac{\partial z_{\gamma}^{l}}{\partial x_{\alpha}^{j}} \frac{1}{n-1} B_{\gamma k l}^{N} \\
- & \left.\frac{\partial z_{\gamma}^{k}}{\partial x_{\alpha}^{i}} \frac{\partial z_{\gamma}^{l}}{\partial x_{\alpha}^{j}} \Pi_{\gamma}^{N_{k l}} \frac{\partial x_{\alpha}^{p}}{\partial z_{\gamma}^{m}} \frac{\partial \log J^{\frac{-1}{n+1}}}{\partial x_{\alpha}^{p}}\right] \xi_{M} \\
+ & {\left[\left(S_{x_{\alpha}}^{z_{\gamma}}\right)_{i j}^{k}+\frac{\partial z_{\gamma}^{p}}{\partial x_{\alpha}^{i}} \frac{\partial z_{\gamma}^{l}}{\partial x_{\alpha}^{j}} \Pi_{\gamma}^{N}{ }^{N} \frac{\partial x_{\alpha}^{k}}{\partial z_{\gamma}^{q}}\right] \frac{\partial}{\partial x_{\alpha}^{k}} . }
\end{aligned}
$$

Using the change of coordinate formulas (1) for $\Pi$ and (8) for $B$, this becomes

$$
\hat{\phi}_{*}^{-1} \nabla_{\hat{\phi}_{*} \frac{\partial}{\partial x_{\alpha}^{2}}}^{\hat{\phi}} \hat{\phi}_{*} \frac{\partial}{\partial x_{\alpha}^{j}}=\frac{1}{n-1} B_{\alpha i j}^{N} \xi_{M}+\Pi_{\alpha i j}^{N^{k}} \frac{\partial}{\partial x_{\alpha}^{k}} .
$$

If we subtract from this

$$
\begin{aligned}
\nabla_{\frac{\partial}{\partial x_{\alpha}^{i}}}^{\hat{M}_{2}} \frac{\partial}{\partial x_{\alpha}^{j}} & =\Gamma^{\hat{M}^{0}}{ }_{i j} \frac{\partial}{\partial x_{\alpha}^{0}}+\Gamma^{\hat{M}^{k}}{ }_{i j} \frac{\partial}{\partial x_{\alpha}^{k}} \\
& =\frac{x_{\alpha}^{0}}{n-1} B_{\alpha}^{M}{ }_{i j} \frac{\partial}{\partial x_{\alpha}^{0}}+\Pi_{\alpha}^{M^{k}}{ }_{i j} \frac{\partial}{\partial x_{\alpha}^{k}} \\
& =\frac{1}{n-1} B_{\alpha}^{M}{ }_{i j} \xi_{M}+\Pi_{\alpha}^{M}{ }^{k}{ }_{i j} \frac{\partial}{\partial x_{\alpha}^{k}}
\end{aligned}
$$

we obtain

$$
\hat{\mathcal{D}}\left(\frac{\partial}{\partial x_{\alpha}^{i}}, \frac{\partial}{\partial x_{\alpha}^{j}}\right)=\frac{1}{n-1}\left(B_{\alpha i j}^{N}-B_{\alpha i j}^{M}\right) \xi_{M}+\left(\Pi_{\alpha i j}^{N^{k}}-\Pi_{\alpha i j}^{M^{k}}\right) \frac{\partial}{\partial x_{\alpha}^{k}} .
$$

Proposition 8.4. $\pi_{*} \hat{\mathcal{D}}\left(\frac{\partial}{\partial x_{\alpha}^{i}}, \frac{\partial}{\partial x_{\alpha}^{j}}\right)$ is defined and equals $\Sigma_{\phi}\left(\frac{\partial}{\partial x_{\alpha}^{i}}, \frac{\partial}{\partial x_{\alpha}^{j}}\right)$.

Proof. Observe that, by the previous proposition, the coefficients of $\partial / \partial x_{\alpha}^{k}$ and of $\xi_{M}$ in $\hat{\mathcal{D}}\left(\frac{\partial}{\partial x_{\alpha}^{i}}, \frac{\partial}{\partial x_{\alpha}^{j}}\right)$ are independent of $x_{\alpha}^{0}$. This means that $\pi_{*} \hat{\mathcal{D}}\left(\frac{\partial}{\partial x_{\alpha}^{i}}, \frac{\partial}{\partial x_{\alpha}^{j}}\right)$ is defined. Since $\pi_{*} \xi_{M}=0$ and $\pi_{*} \frac{\partial}{\partial x_{\alpha}^{k}}=\frac{\partial}{\partial x_{\alpha}^{k}}$,

$$
\pi_{*} \hat{\mathcal{D}}\left(\frac{\partial}{\partial x_{\alpha}^{i}}, \frac{\partial}{\partial x_{\alpha}^{j}}\right)=\left(\Pi_{\alpha i j}^{N^{k}}-\Pi_{\alpha i j}^{M^{k}}\right) \frac{\partial}{\partial x_{\alpha}^{k}}=\Sigma_{\phi}\left(\frac{\partial}{\partial x_{\alpha}^{i}}, \frac{\partial}{\partial x_{\alpha}^{j}}\right) .
$$

We thus obtain a second definition of the Schwarzian derivative of $\phi$ :

Definition 8.2. $\Sigma_{\phi}=\pi_{*} \hat{\mathcal{D}}$.

In the case of a complex projective structure, this second definition may be used to give a very simple proof that $\Sigma_{\phi}=0$ if and only if $\phi$ is complex projective linear when expressed in projective coordinates: 
Theorem 8.5. Let $\Pi^{M}$ and $\Pi^{N}$ be flat. Then $\Sigma_{\phi} \equiv 0$ if and only if for all projective coordinates $z_{\gamma}$ on $N$ and $x_{\alpha}$ on $M, z_{\gamma}=\phi\left(x_{\alpha}\right)$ is a complex projective linear map.

Proof. For projective coordinates $x_{\alpha}$ and $z_{\gamma}, \Pi_{\alpha}^{M^{k}{ }_{i j}}=0$ and $\Pi_{\gamma}^{N_{l p}^{q}}=0$. Also $B_{\alpha i j}^{M}=0$ and $B_{\gamma l p}^{N}=0$. The flatness of $\Pi^{N}$ does not imply $\Pi_{\alpha i j}^{N^{k}}=0$ since $\phi\left(x_{\alpha}\right)$ does not necessarily give projective coordinates. We do have, by the remark following Theorem 7.3,

$$
\nabla_{\frac{\partial}{\partial X_{\alpha}^{I}}}^{\hat{M}^{I}} \frac{\partial}{\partial X_{\alpha}^{J}}=0
$$

for all $I, J$. Therefore, in these coordinates, $\nabla^{\hat{M}}$ is the usual flat connection on $\mathbf{C}^{n+1}$. Of course the same holds for $\nabla^{\hat{N}}$ in the homogeneous coordinates $Z_{\gamma}^{0}=z_{\gamma}^{0}$, $Z_{\gamma}^{i}=z_{\gamma}^{0} x_{\gamma}^{i}$.

If $z_{\gamma}=\phi\left(x_{\alpha}\right)$ is complex projective linear, then

$$
z_{\gamma}^{i}=\frac{a_{j}^{i} x^{j}+b^{i}}{c_{j} x^{j}+d}
$$

If we normalize as usual so that

$$
\operatorname{det}\left(\begin{array}{cc}
a_{j}^{i} & b^{i} \\
c_{j} & d
\end{array}\right)=1
$$

then $z_{\gamma}^{0}=c_{j} x_{\alpha}^{j}+d$, so

$$
Z_{\gamma}^{0}=c_{j} X_{\alpha}^{j}+d X_{\alpha}^{0}, \quad Z_{\gamma}^{i}=a_{j}^{i} X_{\alpha}^{j}+b^{i} X_{\alpha}^{0} .
$$

Then $\hat{\phi}_{*} \frac{\partial}{\partial X_{\alpha}^{I}}$ is a linear combination of $\left\{\frac{\partial}{\partial Z_{\gamma}^{K}}\right\}$, so

$$
\nabla_{\hat{\phi}_{*} \frac{\partial}{\partial X_{\alpha}^{I}}}^{\hat{N}} \hat{\phi}_{*} \frac{\partial}{\partial X_{\alpha}^{J}}=0
$$

and $\hat{\mathcal{D}}\left(\frac{\partial}{\partial X_{\alpha}^{I}}, \frac{\partial}{\partial X_{\alpha}^{J}}\right)=0$ for all $I$ and $J$. Therefore $\hat{\mathcal{D}}=0$ and $\Sigma_{\phi}=\pi_{*} \hat{\mathcal{D}}=0$.

Conversely, if $\Sigma_{\phi}=0$, then $\Pi_{\alpha i j}^{N^{k}}-\Pi_{\alpha i j}^{M^{k}}=0$, and, by the definition of $B$, $B_{\alpha i j}^{N}-B_{\alpha i j}^{M}=0$ also. Thus $\hat{\mathcal{D}} \equiv 0$. This implies

$$
\nabla_{\hat{\phi}_{*} \frac{\partial}{\partial X_{\alpha}^{T}}}^{\hat{N}} \hat{\phi}_{*} \frac{\partial}{\partial X_{\alpha}^{J}}=0
$$

for all $I$ and $J$, which can happen only if $Z_{\gamma}=\hat{\phi}\left(X_{\alpha}\right)$ is affine in $X_{\alpha}$ :

$$
Z_{\gamma}^{I}=A_{J}^{I} X_{\alpha}^{J}+B^{J}
$$

Because $\hat{\phi}$ is a bundle map, $B^{J}$ must equal 0 . Thus $Z_{\gamma}^{0}=A_{J}^{0} X_{\alpha}^{J}, Z_{\gamma}^{I}=A_{J}^{I} X_{\alpha}^{J}$, and so

$$
z_{\gamma}^{i}=\frac{Z_{\gamma}^{i}}{Z_{\gamma}^{0}}=\frac{A_{j}^{i} X_{\alpha}^{j}+A_{0}^{i} X_{\alpha}^{0}}{A_{j}^{0} X_{\alpha}^{j}+Z_{0}^{0} X_{\alpha}^{0}}=\frac{A_{j}^{i} x_{\alpha}^{j}+A_{o}^{j}}{A_{j}^{0} x_{\alpha}^{j}+A_{0}^{0}}
$$




\section{The Schwarzian Derivative and Complex Geodesics}

Finally, we prove the following theorem, which shows the relationship between the Schwarzian derivative as defined in this paper and complex geodesics, thus illuminating how this theory is tied to classical projective geometry.

Let $\Pi$ be a reduced complex projective connection on a complex manifold $M$ of dimension $n>1, \nabla$ an affine connection in the equivalence class associated to $\Pi$ (see Theorem 4.3). From Lemma 4.1, it is clear that all such $\nabla$ have the same complex geodesics. We refer to these as the complex geodesics of $\Pi$.

Theorem 9.1. Let $M$ and $N$ be complex manifolds of the same dimension $n>1$ with reduced holomorphic projective connections $\Pi^{M}$, $\Pi^{N}$. Let $\phi: M \rightarrow N$ be a locally biholomorphic map. Then $\phi$ preserves complex geodesics if and only if $\Sigma_{\phi}=0$.

Proof. Suppose $\Sigma_{\phi}=0$. Let $B$ be a domain in $\mathbf{C}, g: B \rightarrow M$ a complex geodesic, $V=g_{*} \frac{\partial}{\partial z}$. By Lemma 4.1, $\Pi_{V}^{M} V$ is a multiple of $V$ and $\phi \circ g$ is a complex geodesic in $N$ if and only if $\Pi_{\phi_{*} V}^{N} \phi_{*} V$ is a multiple of $\phi_{*} V$. One sees immediately from the first definition of $\Sigma_{\phi}$ that this is the case.

To prove the converse, suppose that $\phi$ maps complex geodesics to complex geodesics. In other words, whenever $g: B \rightarrow M$ is a complex geodesic in $M$, $\phi \circ g: B \rightarrow N$ is a complex geodesic in $N$. In particular, if $g$ is a complex geodesic in $M, V=g_{*} \frac{\partial}{\partial z}$, then $\Sigma_{\phi}(V, V)=\lambda(V) V$ for some differentiable function $\lambda(V)$. A standard existence theorem in the theory of ordinary differential equations implies that given any vector $V_{0} \in T_{P}^{h} M$, there exists a complex geodesic through $P$ such that $V(P)=V_{0}$. Since $\Sigma_{\phi}(V, V)(P)$ depends only on $V(P)$, we may conclude that $\Sigma_{\phi}(X, X)=\lambda(X) X$ for all $V \in T^{h} M ; \lambda$ may be thought of as a complexvalued function on $T^{h} M$. Since $\Sigma_{\phi}$ is a tensor, we have $\lambda(f X)=f \lambda(X)$ for all differentiable functions $f$. We must show $\lambda \equiv 0$. Because $\Sigma_{\phi}$ is a symmetric tensor,

$$
\begin{aligned}
\Sigma_{\phi}(X+Y, X+Y) & =\Sigma_{\phi}(X, X)+2 \Sigma_{\phi}(X, Y)+\Sigma_{\phi}(Y, Y) \\
& =\lambda(X) X+2 \Sigma_{\phi}(X, Y)+\lambda(Y) Y .
\end{aligned}
$$

Also,

$$
\Sigma_{\phi}(X+Y, X+Y)=\lambda(X+Y)(X+Y) .
$$

From (11) and (12), we find

$$
\Sigma_{\phi}(X, Y)=\frac{1}{2}[\lambda(X+Y)-\lambda(X)] X+\frac{1}{2}[\lambda(X+Y)-\lambda(Y)] Y .
$$

Replace $X$ by $2 X$ in (13) to obtain

$$
\Sigma_{\phi}(2 X, Y)=\frac{1}{2}[\lambda(2 X+Y)-2 \lambda(X)] 2 X+\frac{1}{2}[\lambda(2 X+Y)-\lambda(Y)] Y .
$$

By linearity, we also have

$$
\begin{aligned}
\Sigma_{\phi}(2 X, Y) & =2 \Sigma_{\phi}(X, Y) \\
& =[\lambda(X+Y)-\lambda(X)] X+[\lambda(X X+Y)-\lambda(Y)] Y .
\end{aligned}
$$

We may assume that $X$ and $Y$ are linearly independent, so by setting (14) and (15) equal to one another we obtain

$$
\lambda(X+Y)-\lambda(X)=\lambda(2 X+Y)-2 \lambda(X)
$$


and

$$
\frac{1}{2} \lambda(2 X+Y)-\frac{1}{2} \lambda(Y)=\lambda(X+Y)-\lambda(Y) .
$$

Solve (16) and (17) for $\lambda(2 X+Y)$ and set these equal to each other to obtain

$$
\lambda(X+Y)=\lambda(X)+\lambda(Y) .
$$

Finally, put (18) into (13) to obtain

$$
\Sigma_{\phi}(X, Y)=\frac{1}{2} \lambda(Y) X+\frac{1}{2} \lambda(X) Y,
$$

for all $X, Y \in T^{h} M$. To finish the proof, take the trace of both sides of equation (19). This yields

$$
\frac{n+1}{2} \lambda(X)=\operatorname{tr}\left(Y \mapsto \Sigma_{\phi}(Y, X)\right)=0 .
$$

Therefore $\lambda(X)=0$ for all $X$, so by equation (19), $\Sigma_{\phi}=0$.

If $M$ and $N$ are complex manifolds of dimension 1, then, trivially, every locally biholomorphic map from $M$ to $N$ preserves complex geodesics. A differential operator which characterizes complex projective linear transformations in this case must be stronger than one which merely characterizes maps with preserve complex geodesics. This fact sheds light on why the Schwarzian derivative in dimension one looks so different from the Schwarzian derivative in higher dimensions, and, in particular, why it is of order 3 rather than of order 2 .

Corollary (Theorem 4.2). Two affine connections $\nabla$ and $\nabla^{\prime}$ on a complex manifold $M$ are projectively equivalent if and only if they determine the same complex geodesics.

Proof. If $\nabla$ and $\nabla^{\prime}$ are projectively equivalent, then by Lemma 4.1, it is clear that they determine the same complex geodesics.

If conversely, $\nabla$ and $\nabla^{\prime}$ determine the same complex geodesics, then their associated complex projective connections $\Pi$ and $\Pi^{\prime}$ determine the same complex geodesics. Let $\iota:(M, \Pi) \rightarrow\left(M, \Pi^{\prime}\right)$ be the identity map. Theorem 9.1 implies $\Sigma_{\iota}=0$. Therefore $\Pi=\Pi^{\prime}$. By Theorem $4.3, \nabla$ and $\nabla^{\prime}$ are projectively equivalent.

The following is proved as part of a lemma in [12] using methods from algebraic geometry.

Corollary 9.2. If $M$ and $N$ are domains in $\mathbf{C}^{n}$ or in $\mathbf{C} P^{n}$, both with the standard affine connection, then a locally biholomorphic map $\phi: M \rightarrow N$ sends complex lines to complex lines if and only if it is complex projective linear.

Proof. For the standard connection on $\mathbf{C}^{n}$ and for the standard connection on $\mathbf{C P}^{\mathbf{n}}$, the complex lines are the complex geodesics. Theorem 9.1, combined with Theorem 8.5 , yields the result immediately. 


\section{REFERENCES}

[1] E. Cartan, Sur les variétés à connexion projective, Bull. de la Soc. Math. de France 52 (1924), 205-241.

[2] Pierre Deligne, Equations différentielles à points singuliers réguliers, Springer-Verlag, New York, 1970. MR 54:5232

[3] Harley Flanders, The Schwarzian as a curvature, J. Diff. Geom. 4 (1970), 515-519. MR 43:2619

[4] M. Ganesh, Projective structures on compact complex manifolds-geometric theory, Journal of the Indian Math. Soc. 42 (1978), 67-84. MR 82e:5305ba

[5] R. C. Gunning, Special coordinate coverings of Riemann surfaces, Math. Ann. 170 (1967), 67-86. MR 34:7790

[6] __ On uniformization of complex manifolds: the role of connections, Princeton University Press, Princeton, New Jersey, 1978. MR 82e:32034

[7] Masahide Kato, On characteristic forms on complex manifolds, J. of Algebra 138 (1991), 424-439. MR 93b:32042

[8] Shoshichi Kobayashi and Camilla Horst, Topics in complex differential geometry, Complex Differential Geometry, Birkhäuser Verlag, 1983, pp. 4-66. MR 87g:53097

[9] Shoshichi Kobayashi and Tadashi Nagano, On projective connections, J. of Math. and Mech. 13 (1964), 215-235. MR 28:2501

[10] Shoshichi Kobayashi and Takushiro Ochiai, Holomorphic projective structures on compact complex surfaces, Math. Ann. 249 (1980), 75-94. MR 81g:32021

[11] - Holomorphic projective structures on compact complex surfaces. II, Math. Ann. 255 (1981), 519-521. MR 83a:32025

[12] Ngaiming Mok and Sai Kee Yeung, Geometric realizations of uniformization of conjugates of Hermitian locally symmetric manifolds, Complex Analysis and Geometry, Plenum Press, New York, 1993, pp. 253-270. MR 94c:32021

[13] Robert Molzon and Karen Pinney Mortensen, Differential operators associated with holomorphic mappings, To appear in Ann. of Global Analysis and Geom.

[14] Brad Osgood and Dennis Stowe, The Möbius connection in the bundle of conformal 2-jets, Preprint.

[15] - The Schwarzian derivative and conformal mapping of Riemannian manifolds, Duke Math. J. 67 (1992), 57-99. MR 93j:53062

[16] R. R. Simha, The monodromy representations of projective structures, Arch. Math. 52 (1989), 413-416. MR 90g:32010

[17] T.Y. Thomas, On the projective and equi-projective geometry of paths, Proc. Nat. Acad. of Sciences 11 (1925), 199-203.

[18] _ Projective theory of affinely connected manifolds, Math. Zeit. 25 (1926), 723-733.

[19] A.N. Tyurin, On periods of quadratic differentials, Russian Math. Surveys 33 (1978), 169221. MR 81b:14009

[20] H. Weyl, Zur Infinitesimalgeometrie; Einordnung der projecktiven und der konformen Auffassung, Gött. Nach. (1921), 99-112.

[21] J.H.C. Whitehead, The representation of projective spaces, Ann. of Math. 32 (1931), 327-360.

[22] Masaaki Yoshida, Canonical forms of some systems of linear partial differential equations, Proc. Japan Acad. 52 (1976), 473-476. MR 54:13962

[23] _ Orbifold-uniformizing differential equations, Math. Ann. 267 (1984), 125-142. MR 85j:14013

Department of Mathematics, University of Kentucky, Lexington, Kentucky 405060027

E-mail address: molzon@ms.uky.edu

E-mail address: pinney@ms.uky.edu 